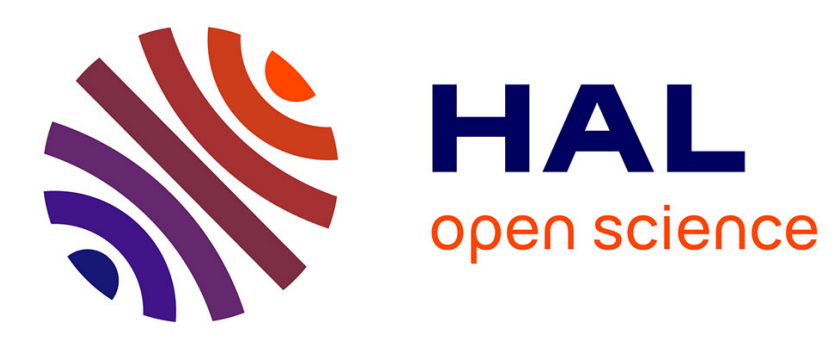

\title{
Recuit laser de cellules solaires fonctionnant sous concentration
}

D. Salles, E. Fogarassy, R. Stuck, P. Siffert

\section{To cite this version:}

D. Salles, E. Fogarassy, R. Stuck, P. Siffert. Recuit laser de cellules solaires fonctionnant sous concentration. Revue de Physique Appliquée, 1980, 15 (4), pp.889-893. 10.1051/rphysap:01980001504088900 . jpa-00244801

\section{HAL Id: jpa-00244801 https://hal.science/jpa-00244801}

Submitted on 1 Jan 1980

HAL is a multi-disciplinary open access archive for the deposit and dissemination of scientific research documents, whether they are published or not. The documents may come from teaching and research institutions in France or abroad, or from public or private research centers.
L'archive ouverte pluridisciplinaire HAL, est destinée au dépôt et à la diffusion de documents scientifiques de niveau recherche, publiés ou non, émanant des établissements d'enseignement et de recherche français ou étrangers, des laboratoires publics ou privés. 


\title{
Recuit laser de cellules solaires fonctionnant sous concentration
}

\author{
D. Salles, E. Fogarassy, R. Stuck et P. Siffert \\ Centre de Recherches Nucléaires, Groupe de Physique et Applications des Semiconducteurs (PHASE) \\ 67037 Strasbourg Cedex, France
}

(Reçu le 3 octobre 1979, révisé le 21 décembre 1979, accepté le 2 janvier 1980)

\begin{abstract}
Résumé. - La résistance série élevée des cellules conventionnelles au silicium rend leur fonctionnement difficile sous concentration. Nous montrons ici que l'irradiation de la couche superficielle diffusée par un faisceau laser pulsé de grande intensité permet de réduire notamment la résistance série, de sorte que des rendements de conversion de $15 \%$ sous 30 soleils et de $12,5 \%$ sous 100 soleils $\left(10 \mathrm{~W} / \mathrm{cm}^{2}\right)$ sont prévus par le calcul.
\end{abstract}

\begin{abstract}
The high series resistance of conventional silicon solar cells reduces noticeably their conversion efficiency when operating under concentration. We demonstrate here that the value of this resistance can be diminished when the diffused surface layer has been irradiated by a high intensity short laser pulse. The expected efficiencies of these cells have been calculated, they range from $15 \%$ to $12.5 \%$ when going from 30 to 100 suns concentration $\left(3-10 \cdot \mathrm{W} / \mathrm{cm}^{2}\right)$.
\end{abstract}

Introduction. - La concentration du rayonnement solaire constitue l'une des méthodes susceptibles d'abaisser le prix de revient de l'électricité d'origine photovoltaïque. La technologie la plus couramment utilisée à l'heure actuelle pour la fabrication de photopiles adaptées aux concentrations moyennes ( $<50$ soleils) étant la diffusion thermique de phosphore dans un substrat de silicium monocristallin de type $\mathrm{P}$, nous avons restreint notre étude à cette méthode.

Les performances des photopiles à concentration sont en majeure partie limitées par l'importance de leur résistance série interne. En effet, en utilisant un modèle mathématique simple, Fabre [1] a montré que le rendement de conversion d'une photopile décroît rapidement lorsque sa résistance série augmente. Nous avons reporté sur la figure 1 l'évolution du rendement de conversion, calculée de manière théorique par cet auteur, en fonction de la concentration pour une photopile de $2 \times 2 \mathrm{~cm}^{2}$ réalisée sur silicium de type $\mathrm{P}$, maintenue à une température de $300 \mathrm{~K}$. On notera que la résistance série doit dans ce cas être inférieure à $30-40 \mathrm{~m} \Omega$ si l'on veut conserver sous concentration moyenne ( $\times 50$ soleils) un rendement de conversion au moins égal à celui mesuré sous AM1.

La résistance série dépend de nombreux paramètres, dont la résistivité du matériau de base, la résistance carrée de la couche diffusée superficielle, les résistances de contact, les résistances des métallisations et la géométrie de la grille de la face avant. Néanmoins

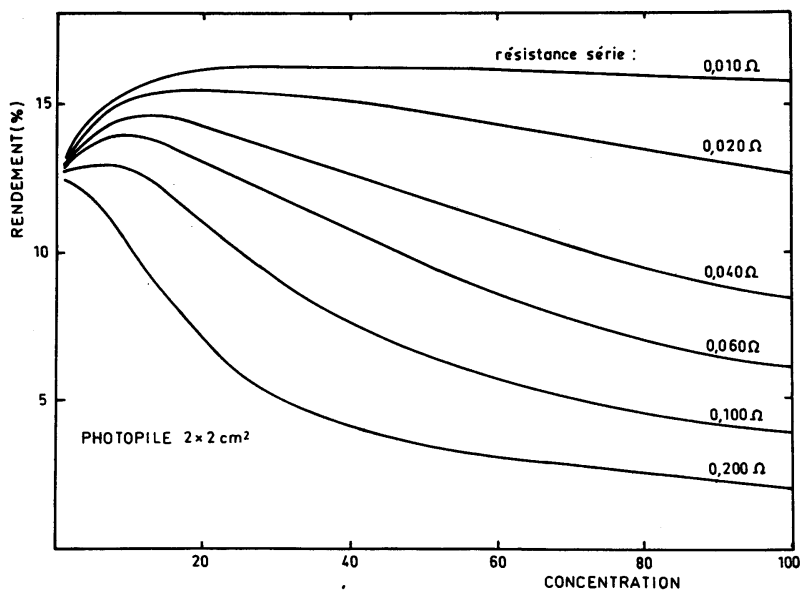

Fig. 1. - Evolution du rendement d'une cellule solaire au silicium à jonction $\mathrm{N}^{+} / \mathrm{P}$ de $4 \mathrm{~cm}^{2}$ de surface en fonction de la concentration du rayonnement solaire pour différentes valeurs de la résistance série.

[Conversion efficiency of a $4 \mathrm{~cm}^{2}$ silicon $\mathrm{N}^{+} / \mathrm{P}$ junction cell as a function of sun concentration and for various values of the series resistance.]

il a été montré $[1,2]$ que la résistance série d'une cellule conventionnelle (cellule au silicium monocristallin à jonction $\mathrm{N}^{+}-\mathrm{P}$ de $2 \times 2 \mathrm{~cm}^{2}$ réalisée sur un substrat de résistivité $1,5 \Omega \mathrm{cm}$ et d'épaisseur $300 \mu \mathrm{m})$ dépend en majeure partie de la résistance de la couche superficielle (généralement caractérisée par sa résistance carrée $R_{\square}$ égale à $\rho / e$, où $\rho$ est la 
résistivité de cette couche, $e$ son épaisseur) et en second lieu de la résistance du contact ohmique arrière (ces deux résistances peuvent atteindre $0,2 \Omega$ alors que les autres sont toutes inférieures à $10 \mathrm{~m} \Omega$ ). S'il est relativement aisé de diminuer la résistance du contact ohmique, il est plus difficile d'agir sur $R_{\square}$. En effet, la technique de diffusion thermique classique ne permet pas d'obtenir des concentrations de phosphore électriquement actif supérieures à $2 \times 10^{20} \mathrm{~cm}^{-3}$, ce qui correspond, pour une profondeur de jonction de l'ordre de $4000 \AA$, à des valeurs de $R_{\square}$ supérieures à $35 \Omega$.

Puisque l'on ne peut réduire $R_{\square}$ par des techniques de dopage classiques, une première méthode pour diminuer la résistance série $R_{\mathrm{s}}$ consiste à minimiser l'effet de $R_{\square}$ sur $R_{\mathrm{s}}$ en utilisant des grilles de collecte dont la géométrie est conçue de manière à réduire le parcours, dans la couche de surface, des porteurs photogénérés avant leur collection [1].

Nous proposons dans cet article une autre méthode qui consiste à agir directement sur $R_{\square}$, utilisant une irradiation laser brève. En effet, on a montré récemment qu'il est possible de diminuer la résistance carrée d'une couche de silicium dopée au phosphore [3] en augmentant la concentration de dopant électriquement actif grâce à une irradiation laser convenable.

Appliquée à une cellule diffusée conventionnelle, cette technique permet de diminuer $R_{\square}$ sans pour autant entraîner un accroissement sensible de la profondeur de jonction qui dégraderait la réponse spectrale de la cellule dans les courtes longueurs d'onde.

Nous avons ensuite évalué, à l'aide d'un modèle simple, la résistance série puis le rendement que l'on peut s'attendre à obtenir d'une telle photopile, traitée par laser avant dépôt de la métallisation, pour diverses géométries de grille.

1. Effets sur $\boldsymbol{R}_{\square}$ d'une irradiation laser. - 1.1 LIMITES DE DOPAGE PAR DIFFUSION THERMIQUE CONVENTIONNELLE. - A l'heure actuelle, la plupart des cellules sont réalisées à partir de silicium monocristallin de faible résistivité, dans lequel la jonction $\mathrm{N}^{+}$-P est réalisée par diffusion thermique de phosphore à $850^{\circ} \mathrm{C}$ environ sur une profondeur de $0,4 \mu \mathrm{m}$. Dans ces conditions, la concentration totale de phosphore introduite est donnée par la solubilité limite de cet élément dans le silicium, soit $5 \times 10^{20} \mathrm{~cm}^{-3}$, pour cette température de diffusion. On constate cependant que la concentration de dopants électriquement actifs ne peut dépasser $2 \times 10^{20} \mathrm{~cm}^{-3}$. Tout le phosphore en excès est inactif, soit parce qu'il précipite, soit parce qu'il est associé à des lacunes doublement chargées (pairage) [4]. Du fait de cette limite les valeurs de $R_{\square}$ obtenues dépassent généralement $35 \Omega$, si la profondeur de jonction n'excède pas $0,4 \mu$.

1.2 EFFETS D'UNE IMPULSION LASER BRÈVE SUR LA ZONE DIFFUSÉE. - 1.2.1 Conditions expérimentales.
- Dans une première étape, des jonctions $\mathbf{N}^{+} / \mathbf{P}$ ont été réalisées par diffusion de phosphore à partir d'une source de $\left(\mathrm{POCl}_{3}\right)$ gazeuse à $875^{\circ} \mathrm{C}$ pendant $40 \mathrm{~min}$ sur des plaquettes $(\varnothing 57 \mathrm{~mm})$ de silicium monocristallin $\langle 111\rangle$ Czochralski de faible résistivité $(1,5 \Omega . \mathrm{cm})$, de $300 \mu \mathrm{m}$ d'épaisseur [5]. La jonction a ensuite été irradiée par une impulsion lumineuse issue d'un laser à rubis $(\lambda=0,6943 \mu \mathrm{m})$ de 20-35 ns, pour une densité d'énergie variant de 0,8 à $2 \mathrm{~J} / \mathrm{cm}^{2}$. Les couches ont été caractérisées par SIMS, par RBS, et les mesures de résistance carrée ont été effectuées par la méthode des 4 pointes.

1.2.2 Résultats. - Nous nous limiterons ici à l'effet sur $R_{\square}$ du traitement laser de la jonction. Les résultats expérimentaux, reportés sur la figure 2 , montrent qu'il est possible de réduire notablement la résistance carrée de la couche de surface; en effet des résistances carrées de $12 \Omega$ ont pu être atteintes pour une irradiation laser de $2 \mathrm{~J} / \mathrm{cm}^{2}$.

Il existe à l'heure actuelle différents modèles qui expliquent le processus de transfert de l'énergie du faisceau laser absorbée par le silicium; on peut admettre cependant que tout se passe comme si cette énergie absorbée pendant 30 ns sur 1000 à $2000 \AA$ était suffisante pour entraîner la fusion de silicium jusqu'à une profondeur de 3000 à $5000 \AA$, fonction de la densité d'énergie du pulse laser, sa recristallisation en épitaxie liquide intervenant très rapidement ( $100 \mathrm{~ns})$ [6]. Dans ces conditions, les précipités de phosphore créés en surface lors de la diffusion thermique sont dissous, et la solidification intervient ensuite trop rapidement pour permettre la formation de nouvelles associations ou la migration du dopant vers des sites de précipitation. Il en résulte un accroissement de la

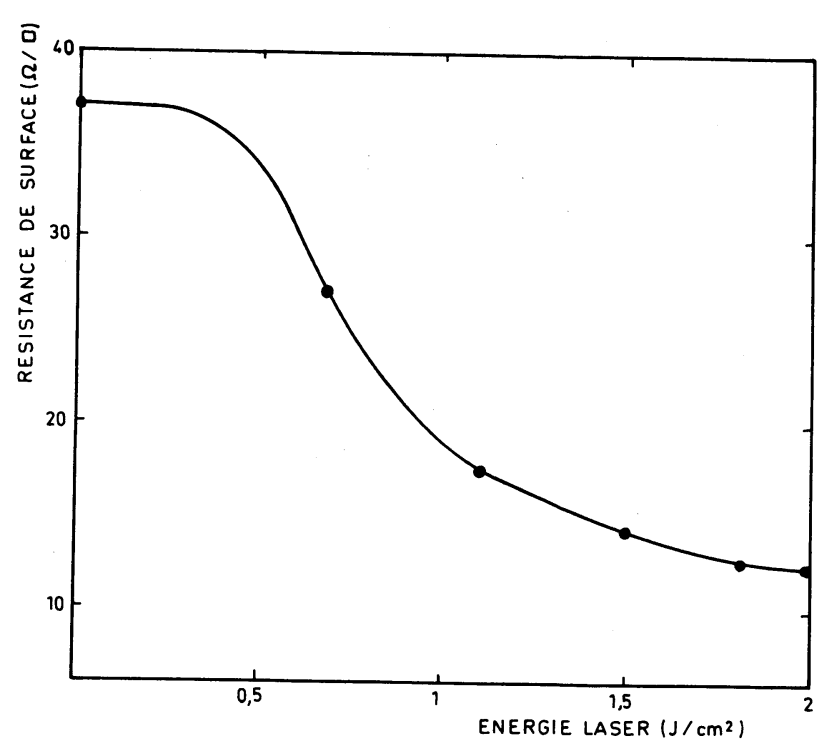

Fig. 2. - Evolution de la résistance carrée $R_{\mathrm{a}}$ de la couche superficielle $\mathrm{N}^{+}$d'une jonction $\mathrm{N}^{+} / \mathrm{P}$ diffusée en fonction de l'énergie de l'impulsion laser de recuit.

[Sheet resistance $R_{\square}$ change of the surface $\mathrm{N}^{+}$region of a diffused $\mathrm{N}^{+} / \mathrm{P}$ junction $v s$. laser energy used for annealing.] 
concentration de dopants électriquement actif qui se traduit par une diminution de $R_{\square}$.

Dans les conditions de recuit laser utilisées (densité d'énergie $\leqslant 2 \mathrm{~J} / \mathrm{cm}^{2}$ ), la profondeur de jonction reste inférieure à $5000 \AA$ [6], c'est-à-dire du même ordre que celle obtenue par diffusion thermique ; la réduction de $R_{\square}$ est donc essentiellement due au processus de réactivation des dopants.

Le tableau suivant montre que la diminution de la résistance série, consécutive au traitement laser s'accompagne d'une amélioration des performances, testées sous des conditions AM1, d'une cellule standard RTC ( $\varnothing 57 \mathrm{~mm})$.

\begin{tabular}{lccccc}
\multicolumn{1}{c}{ Pile } & $\begin{array}{c}I_{\mathrm{SC}} \\
(\mathrm{mA})\end{array}$ & $\begin{array}{c}V_{\text {oc }} \\
(\mathrm{mV})\end{array}$ & F.F. & $\eta(\%)$ & $R_{\mathrm{S}}(\Omega)$ \\
$\begin{array}{l}\text { Standard } \\
\text { Traitée au laser } \\
\left(1,1 \mathrm{~J} / \mathrm{cm}^{2}\right)\end{array}$ & - & - & - & - & - \\
& 690 & 590 & 0,72 & 11,8 & 0,06 \\
& 710 & 600 & 0,75 & 12,8 & 0,04
\end{tabular}

\section{On relève notamment :}

- un accroissement de la tension en circuit ouvert de 10 à $20 \mathrm{mV}$, par suite de l'augmentation de la concentration de dopants actifs dans la couche de surface ;

- une élévation du courant de court-circuit due à l'amélioration des paramètres optiques de la couche superficielle par suite de la dissolution des précipités.

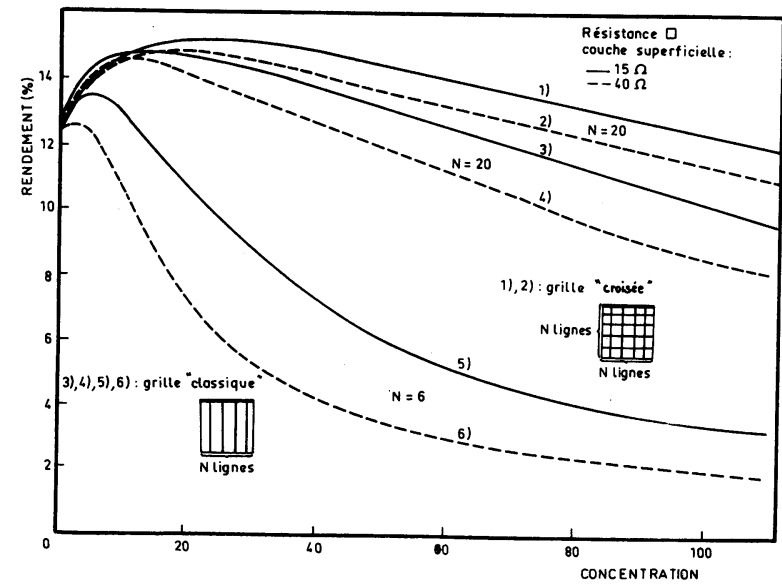

Fig. 3. - Evolution du rendement de cellules solaires au silicium à jonction $\mathrm{N}^{+} / \mathrm{P}$ ayant subi $\left(R_{\mathrm{a}}=15 \Omega\right)$ ou non $\left(R_{\mathrm{a}}=40 \Omega\right)$ un recuit par faisceau laser pulsé, en fonction de la concentration et pour différents types de grilles.

$\left[\mathrm{N}^{+} / \mathrm{P}\right.$ silicon solar cell efficiency $v s$. sun concentration before $\left(R_{\mathrm{a}}=40 \Omega\right)$ and after $\left(R_{\mathrm{a}}=15 \Omega\right)$ laser annealing for various kind of grids.]

2. Calcul théorique de la résistance série en fonction de $\boldsymbol{R}_{\square}$. - Plusieurs modèles de calcul de la résistance série $R_{\mathrm{S}}$ d'une cellule à jonction $\mathrm{P}-\mathrm{N}$ ont déjà été proposés dans la littérature $[2,7,8]$. Nous avons bâti notre modèle suivant le principe des zones unités utilisé par Handy [8], en y apportant certaines modifications. D’autre part, la géométrie de la grille intervenant dans le calcul de $R_{\mathrm{S}}$, nous avons pris en considération deux types de grille, une grille classique, déjà étudiée par Handy, et une grille croisée.

\subsection{CAlCUl DE LA RÉSiSTANCE SÉRIE D'UNE CELlUle} MUNIE D'UNE GRILLE « CLASSIQUE ». - Nous appellerons grille classique une grille dont la géométrie est représentée sur la figure $4 a$; la symétrie qu'elle présente permet d'introduire le concept de zones unités [8].

(a)

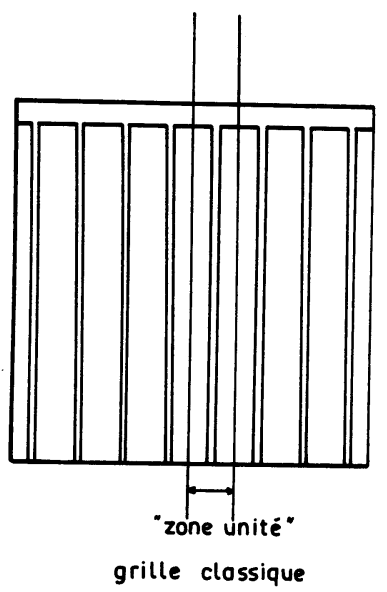

Fig. 4a. - Définition de la zone unité [8] sur une cellule munie d'une grille classique.

[Definition of the unit area [8] of a cell having a conventional grid.]

$R_{\mathrm{s}}$ peut se mettre sous la forme de la somme des résistances suivantes :

- résistance de la métallisation arrière;

- résistance de contact métallisation arrièresubstrat ;

- résistance du matériau de base;

$-R_{\mathrm{N}}$

où $R_{\mathrm{N}}$ est une résistance qui correspond au trajet des porteurs photogénérés depuis la jonction, à travers la couche de surface, puis à travers la grille, jusqu'au circuit extérieur. Le calcul le plus délicat est celui de la résistance $R_{\mathrm{NS}}$ correspondant au trajet des porteurs dans la couche superficielle. Nous ne tiendrons compte dans le calcul que du déplacement de porteurs parallèle à la surface de la cellule (le trajet perpendiculaire à la surface correspond à une résistance négligeable, inférieure à $10^{-7} \Omega$ ).

Du fait de la symétrie, $R_{\mathrm{NS}}$ peut être calculée à partir de la résistance équivalente $R_{\mathrm{NS}_{0}}$ d'une zone unité.

Pour calculer $R_{\mathrm{NS}_{0}}$, on fait l'approximation que le trajet dans la couche superficielle des porteurs photogénérés se fait suivant deux directions (Fig. 4b) :

Ox pour ceux créés dans la zone 1 ;

Oy pour ceux créés dans la zone 2 . 


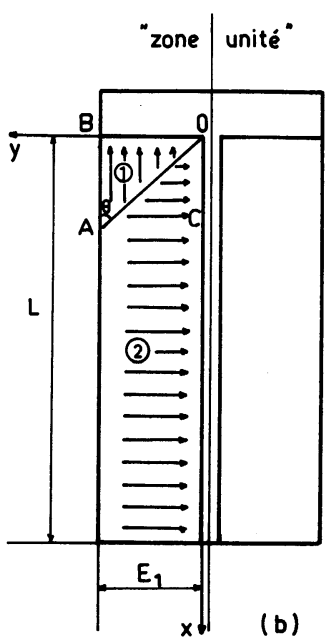

Fig. 4b. - Trajet des porteurs photogénérés dans la zone unité d'une cellule munie d'une grille classique.

[Displacement of the photogenerated carriers in an unit area with a conventional grid.]

La position du point A (Fig. 4b) est déterminée par l'équation :

$$
V_{\mathrm{A}}-V_{\mathrm{B}}=V_{\mathrm{A}}-V_{\mathrm{C}} .
$$

La résolution de cette équation permet de déterminer langle $\theta$.

Compte tenu de ces hypothèses, nous avons calculé respectivement les résistances $R_{1}$ et $R_{2}$ des régions (1) et (2). Nous ne détaillerons ici que le calcul de $R_{1}$.

Pour une densité de photons incidents $\Phi_{\mathrm{i}}$, le courant $I(r)$ qui traverse la surface $A(r)$ (Fig. 5) correspond à des porteurs créés par l'éclairement de la surface $A_{\mathrm{i}}(r)$. D'où :

$$
I(r)=\eta \cdot A_{\mathrm{i}}(r) \Phi_{\mathrm{i}},
$$

où $\eta$ représente le rendement quantique.

En supposant que les lignes de champs entre les surfaces $A(r)$ et $A(r+\mathrm{d} r)$ sont parallèles à l'axe $A r$, le champ électrique à travers $A(r)$ s'écrira :

$$
\mathbf{E}(r)=\rho . J(r) . \mathbf{i}
$$

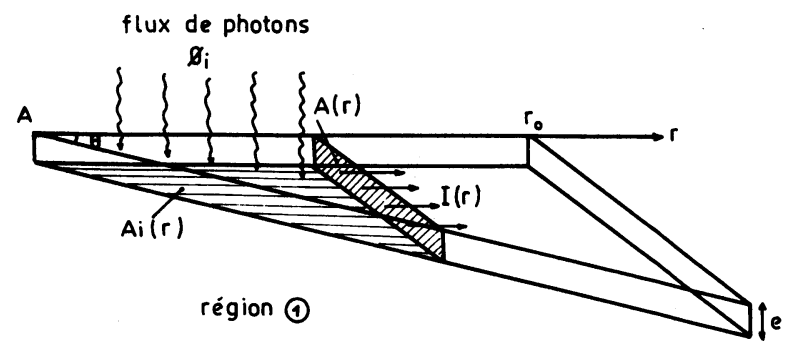

Fig. 5. - Notations utilisées dans le calcul de la résistance de la région (1) d'une zone unité pour une cellule munie d'une grille classique.

[Definition of the various terms for the calculation of the resistance of the region (1) of an unit area.] où $J(r)$ désigne la densité de courant qui traverse $A(r), \rho$ la résistivité de la couche.

La chute du potentiel entre $A(r)$ et $A(r+\mathrm{d} r)$ s'écrit alors :

$$
\begin{aligned}
& V(r+\mathrm{d} r)-V(r)=\mathrm{d} V(r)=-\mathbf{E}(r) . \mathbf{i} \mathrm{d} r= \\
&=-\rho \eta \frac{A_{\mathrm{i}}(r)}{A(r)} \cdot \Phi_{\mathrm{i}} .
\end{aligned}
$$

La chute du potentiel entre 0 et $r_{0}$ sera :

$$
V_{0}-V_{r_{0}}=-\int_{r_{0}}^{0} \rho \cdot \eta \cdot \frac{A_{\mathrm{i}}(r)}{A(r)} \cdot \Phi_{\mathrm{i}} \cdot \mathrm{d} r
$$

sachant que

$$
A_{\mathrm{i}}(r)=\frac{r^{2}}{2} \operatorname{tg} \theta \quad \text { et } \quad A(r)=e \cdot r \cdot \operatorname{tg} \theta
$$

on aura :

$$
V_{0}-V_{r_{0}}=\frac{\rho \eta \Phi_{i} r_{0}^{2}}{4 e}
$$

$R_{1}$ s'écrit donc :

$$
\begin{gathered}
R_{1}=\frac{V_{0}-V_{r_{0}}}{I_{\text {tot }}} \\
I_{\text {tot }}=\eta \Phi_{\mathrm{i}}\left(A_{\mathrm{i}}(r)\right)_{r=r_{0}}=\frac{\eta \Phi_{\mathrm{i}} r_{0}^{2} \operatorname{tg} \theta}{2}
\end{gathered}
$$

d'où

$$
R_{1}=\rho \frac{\operatorname{cotg} \theta}{2 e}=\frac{R_{\square}}{2} \operatorname{cotg} \theta \quad \text { avec } \quad R_{\square}=\frac{\rho}{e} .
$$

Le calcul de $R_{2}$, fondé sur le même principe, aboutit à :

$$
\begin{aligned}
R_{2}= & \frac{R_{\square}}{2}[\operatorname{tg} \theta- \\
& \left.-\frac{\left(L \operatorname{tg} \theta-E_{1}\right)^{2} \cdot \log e\left(L /\left(L-E_{1} \operatorname{cotg} \theta\right)\right)}{E_{1} \cdot\left(L-E_{1} \operatorname{cotg} \theta / 2\right)}\right] .
\end{aligned}
$$

Un algorithme permet ensuite de déterminer $R_{\mathrm{NS}_{0}}$, puis $R_{\mathrm{S}}$, à partir de $R_{1}$ et $R_{2}$ et des autres résistances entrant en ligne de compte. Le calcul montre que $R_{1}$ et $R_{2}$, donc $R_{\mathrm{S}}$ ne dépendent de $\rho$ et $e$ que par le rapport $\rho / e=R_{\square}$.

2.2 CALCUl De LA RÉsistaNCE SÉRIE D'UNE CELLULE MUNIE D'UNE GRILLE " CROISÉE 》. - La géométrie de la grille croisée que nous avons étudiée est représentée sur la figure 3. L'intérêt de cette géométrie est de permettre d'atteindre des résistances série plus faibles qu'avec une grille classique, grâce à la réduction du parcours moyen des porteurs photogénérés avant leur collection par la métallisation, tout en conservant un taux de couverture raisonnable $(<10 \%)$.

Le calcul de la résistance série d'une cellule munie 
d'une telle grille procède du même principe que dans le cas d'une grille classique, en tenant compte des symétries propres à sa géométrie.

Les valeurs des paramètres intervenant dans le calcul de $R_{\mathrm{S}}$, pour les deux types de grilles étudiées, sont les suivantes :

— côté de la cellule : $2 \mathrm{~cm}$;

- épaisseur de la cellule : $300 \mu \mathrm{m}$;

- largeur de la barre collectrice : $500 \mu \mathrm{m}$;

- largeur des lignes de la grille : $40 \mu \mathrm{m}$;

— résistivité matériau de base : $1 \Omega . \mathrm{cm}$;

- résistance carrée de la métallisation :

$$
5 \times 10^{-3} \Omega \text {; }
$$

$-R_{\square}: 15 \Omega$ ou $40 \Omega$;

- nombre de lignes de la grille : 6 ou 20 ;

- taux de couverture maximum $(N=20$, grille croisée) : $10 \%$.

2.3 COMPARAISON DES RENDEMENTS DE CONVERSION THÉORIQUES DES DIFFÉRENTES CELLULES. - Le rendement de conversion d'une photopile sous un éclairement donné peut se calculer de manière théorique à partir de sa relation caractéristique $I(V)$. Lorsque l'on tient compte de la résistance série, cette relation est couramment admise sous la forme implicite suivante :

$$
I=I_{0}\left(\exp q \frac{\left(V-R_{\mathrm{S}} . I\right)}{n k T}-1\right)-I \mathrm{ph}
$$

$I_{0}$ est le courant de saturation, Iph le photocourant, $R_{\mathrm{S}}$ la résistance série.

Nous avons représenté sur la figure 3 l'évolution $\mathrm{du}$ rendement de conversion théorique en fonction de la concentration, pour des cellules dotées des deux types de grille étudiés plus haut.

Dans les cas des cellules à jonction P-N réalisées par des techniques conventionnelles (pour lesquelles
$R_{\square}$ est de l'ordre de $40 \Omega$ ) on voit l'intérêt d'une grille croisée par rapport à une grille classique, même dans le cas où cette dernière contient un nombre de lignes plus grand. Le nombre de lignes ne peut cependant pas être trop grand, car le taux de couverture devient alors trop important et le rendement chute d'autant, à moins de diminuer la largeur des lignes pour conserver un taux de couverture raisonnable $(\leqslant 10 \%)$. Malheureusement, on peut difficilement descendre en dessous de largeurs de l'ordre de $40 \mu \mathrm{m}$ pour des raisons technologiques; de plus, la diminution de la largeur des lignes jusqu'à des valeurs relativement faibles entraîne une augmentation de la résistance de ces lignes qui peut alors devenir conséquente et nuire à la résistance série.

Par contre, si l'on fait subir un recuit par faisceau laser pulsé à une cellule à jonction P-N avant dépôt de la métallisation, quelle que soit la méthode utilisée pour réaliser la jonction (diffusion thermique classique, techniques d'implantation ou d'effluvage...), on peut obtenir des performances intéressantes sous concentration avec une grille classique, à condition que $\mathbf{N}$ soit suffisamment grand (rendement maximal de $14,5 \%$ sous 15 soleils). Une grille croisée donne évidemment des résultats meilleurs mais la différence avec une grille classique est moins sensible sur une cellule qui a subi le traitement laser (le rendement maximum dans ce cas est de l'ordre de $15 \%$ sous 30 soleils et supérieur à $12,5 \%$ sous 100 soleils, la cellule étant maintenue à une température de $300 \mathrm{~K}$ ).

3. Conclusion. - Nous avons montré que l'intervention du recuit laser dans le processus de fabrication d'une cellule solaire au silicium permet d'en améliorer les performances sous concentration et d'envisager leur utilisation sous des concentrations plus élevées (jusqu'à 100 soleils) avec des rendements acceptables; si l'on utilise une telle technologie le recours à une grille sophistiquée généralement coûteuse n'est plus indispensable.

\section{Bibliographie}

[1] Fabre, E., De Smet, L., Mertens, R., Proc. of the International Conf., Luxembourg (27-30.9.1977), p. 249, ed. D. Reidel.

[2] Chambouleyron, I., Proc. of the International Conf., Luxembourg (27-30.9.1977), p. 987, ed. D. Reidel.

[3] Fogarassy, E., Stuck, R., Muller, J. C., A. and Grob, J. J., SifferT, P., 21 th Electronic Materials Conference June 1979, Boulder, à paraître J. Electron. Mater.
[4] FaIR, R. B. and TSAI, J. C. C., J. Electrochem. Soc. 124 (1977) 1109.

[5] Echantillons mis à notre disposition par SALles, Y., RTC, Caen.

[6] Bell, R. O., Toulemonde, M., Siffert, P., Appl. Phys. 19 (1979) 313.

[7] Wolf, M., Proc. of the I.R.E. 48 (1960) 1246.

[8] HaNDy, R. J., Solid State Electron. 10 (1967) 765. 